\title{
COHEN-MACAULAY BIPARTITE GRAPHS IN ARBITRARY CODIMENSION
}

\author{
HASSAN HAGHIGHI, SIAMAK YASSEMI, AND RAHIM ZAARE NAHANDI
}

(Communicated by Irena Peeva)

\begin{abstract}
Let $G$ be an unmixed bipartite graph of dimension $d-1$. Assume that $K_{n, n}$, with $n \geq 2$, is a maximal complete bipartite subgraph of $G$ of minimum dimension. Then $G$ is Cohen-Macaulay in codimension $t$ if and only if $t \geq d-n+1$. This is derived from a characterization of CohenMacaulay bipartite graphs by Herzog and Hibi and generalizes a recent result of Cook and Nagel on unmixed Buchsbaum graphs. Furthermore, we show that any unmixed bipartite graph $G$ which is Cohen-Macaulay in codimension $t$, is obtained from a Cohen-Macaulay graph by replacing certain edges of $G$ with complete bipartite graphs. Thus, in light of combinatorial characterization of Cohen-Macaulay bipartite graphs, our result may be considered purely combinatorial.
\end{abstract}

\section{INTRODUCTION}

Cohen-Macaulay simplicial complexes are among central research topics in combinatorial commutative algebra. While characterization of such complexes is a far reaching problem, one appeals to study specific families of Cohen-Macaulay simplicial complexes. Flag complexes are among important families of complexes recommended to study [10, page 100]. However, it is known that a simplicial complex is Cohen-Macaulay if and only if its barycentric subdivision is a Cohen-Macaulay flag complex. Therefore, a characterization of Cohen-Macaulay flag complexes is equivalent to a characterization of Cohen-Macaulay simplicial complexes. Nevertheless, after all, the ideal of a flag complex is generated by quadratic square-free monomials, which are simpler compared to arbitrary square-free monomial ideals. Furthermore, it seems that, expressing many combinatorial properties in terms of graphs is more convenient. As some evidences, the characterization of unmixed bipartite graphs by Villarreal [11] and Cohen-Macaulay bipartite graphs by Herzog and Hibi [5] are well expressed in terms of graphs.

On the other hand, in the hierarchy of families of graphs with respect to CohenMacaulay property, Buchsbaum complexes appear right after Cohen-Macaulay ones. Unmixed bipartite Buchsbaum graphs were characterized by Cook and Nagel [1] (also by the authors [3]). Natural families of graphs in this hierarchy are bipartite $\mathrm{CM}_{t}$ graphs, namely, graphs for which the independence complex is pure and Cohen-Macaulay in codimension $t$. The concept of $\mathrm{CM}_{t}$ simplicial complexes was introduced in [4] which is the pure version of simplicial complexes Cohen-Macaulay in codimension $t$ studied by Miller, Novik and Swartz [6]. In this note, we give

Received by the editors March 19, 2013 and, in revised form, December 15, 2013.

2010 Mathematics Subject Classification. Primary 13H10, 05 C75.

Key words and phrases. Flag complex, Cohen-Macaulay complex in codimension. 
characterizations of unmixed bipartite $\mathrm{CM}_{t}$ graphs in terms of its dimension and the minimum dimension of its maximal nontrivial complete bipartite subgraphs. Cook and Nagel showed that the only non-Cohen-Macaulay unmixed Buchsbaum bipartite graphs are complete bipartite graphs [1, Theorem 4.10] and [3. Theorem 1.3]. We give a generalization of this fact to unmixed bipartite graphs which are Cohen-Macaulay in arbitrary codimension. In the next section we gather necessary definitions and known results to be used in the rest of the paper. In Section 3 we improve some results on joins of simplicial complexes and disjoint unions of graphs with respect to the $\mathrm{CM}_{t}$ property. Section 4 is devoted to two characterizations of bipartite $\mathrm{CM}_{t}$ graphs and some examples.

\section{Preliminaries}

For basic definitions and general facts on simplicial complexes we refer to the book of Stanley [10]. By a complex we will always mean a simplicial complex. Let $G=(V, E)$ be a simple graph with vertex set $V$ and edge set $E$. The inclusive neighborhood of $v \in V$ is the set $N[v]$ consisting of $v$ and vertices adjacent to $v$ in $G$. The independence complex of $G=(V, E)$ is the complex $\operatorname{Ind}(G)$ with vertex set $V$ and with faces consisting of independent sets of vertices of $G$, i.e., sets of vertices of $G$ where no two of them are adjacent. These complexes are called flag complexes, and their Stanley-Reisner ideal is generated by quadratic square-free monomials. By dimension of a graph $G$ we mean the dimension of the complex $\operatorname{Ind}(G)$ (= independence number of $G-1)$. A graph $G$ is said to be unmixed if $\operatorname{Ind}(G)$ is pure.

For an integer $t \geq 0$, a complex $\Delta$ is called $\mathrm{CM}_{t}$ if it is pure and for every face $F \in \Delta$ with $\#(F) \geq t, \operatorname{link}_{\Delta}(F)$ is Cohen-Macaulay. This is the same as pure complexes which are Cohen-Macaulay in codimension $t$. Accordingly, $\mathrm{CM}_{0}$ and $\mathrm{CM}_{1}$ complexes are precisely Cohen-Macaulay and Buchsbaum complexes, respectively. Clearly, a $\mathrm{CM}_{t}$ complex is $\mathrm{CM}_{r}$ for all $r \geq t$ and a complex of dimension $d-1$ is always $\mathrm{CM}_{d-1}$. One uses the convention that for $t<0, \mathrm{CM}_{t}$ would mean $\mathrm{CM}_{0}$. A graph $G$ is called $\mathrm{CM}_{t}$ if $\operatorname{Ind}(G)$ is $\mathrm{CM}_{t}$. A basic tool for checking $\mathrm{CM}_{t}$ property of complexes is the following lemma.

Lemma 2.1 (4 Lemma 2.3]). Let $t \geq 1$ and let $\Delta$ be a nonempty complex. Then the following are equivalent:

(i) $\Delta$ is a $\mathrm{CM}_{t}$ complex.

(ii) $\Delta$ is pure and link $k_{\Delta}(v)$ is $\mathrm{CM}_{t-1}$ for every vertex $v \in \Delta$.

By the straightforward identity $\operatorname{link}_{\operatorname{Ind}(G)}(v)=\operatorname{Ind}(G \backslash N[v])$, the counterpart of this lemma for graphs is the following:

Lemma 2.2. Let $t \geq 1$ and let $G$ be a graph. Then the following are equivalent:

(i) $G$ is a $\mathrm{CM}_{t}$ graph.

(ii) $G$ is unmixed and $G \backslash N[v]$ is a $\mathrm{CM}_{t-1}$ graph for every vertex $v \in G$.

We recall some basic relevant facts on bipartite graphs. A graph $G=(V, E)$ is called bipartite if $V$ is a disjoint union of a partition $V_{1}$ and $V_{2}$ such that $E \subset V_{1} \times V_{2}$. If $\#\left(V_{1}\right)=m$ and $\#\left(V_{2}\right)=n$ and $E=V_{1} \times V_{2}$, then $G$ is the complete bipartite graph $K_{m, n}$. We will be interested in unmixed complete bipartite graphs $K_{n, n}$.

Unmixed bipartite graphs are characterized by Villarreal in the following result. 
Theorem 2.3 ([11, Theorem 1.1]). Let $G$ be a bipartite graph without an isolated vertex. Then $G$ is unmixed if and only if there is a partition $V_{1}=\left\{x_{1}, \cdots, x_{n}\right\}$ and $V_{2}=\left\{y_{1}, \cdots, y_{n}\right\}$ of vertices of $G$ such that

(1) $x_{i} y_{i}$ is an edge in $G$ for $1 \leq i \leq n$ and

(2) if $x_{i} y_{j}$ and $x_{j} y_{k}$ are edges in $G$, for some distinct $i, j$ and $k$, then $x_{i} y_{k}$ is an edge in $G$.

In this case, such a partition and ordering is called a pure order of $G$. The edges $x_{i} y_{i}, i=1, \cdots, n$ are called perfect matching edges of $G$. A pure order is said to have a cross if, for some $i \neq j, x_{i} y_{j}$ and $x_{j} y_{i}$ are both edges in $G$. Otherwise, the order is called cross-free (see [1, § 4]). For unmixed bipartite graphs, being cross-free is independent from an ordering of vertices of $G$. More precisely, if $G$ has a cross in some pure ordering, it has a cross in every pure ordering [1, Lemma 4.5].

An immediate consequence of Theorem 2.3 is the following useful lemma.

Lemma 2.4. Let $G$ be an unmixed bipartite graph with pure order of vertices $\left(\left\{x_{1}, \cdots, x_{d}\right\},\left\{y_{1}, \cdots, y_{d}\right\}\right)$ and let $K_{n, n}$ be a complete bipartite subgraph of $G$ on $\left(\left\{x_{i_{1}}, \cdots, x_{i_{n}}\right\},\left\{y_{i_{1}}, \cdots, y_{i_{n}}\right\}\right)$.

(i) If $x_{j} y_{i_{k}}$ is an edge in $G$ for some $j$ and $k$, then $x_{j} y_{i_{l}}$ is an edge in $G$ for all $l=1, \cdots, n$.

(ii) If $x_{i_{k}} y_{j}$ is an edge in $G$ for some $k$ and $j$, then $x_{i_{l}} y_{j}$ is an edge in $G$ for all $l=1, \cdots, n$.

Proof. The assertion (i) is immediate by Theorem 2.3 because $x_{i_{k}} y_{i_{l}}$ is an edge in $K_{n, n} \subset G$ for all $l=1, \cdots, n$. Also (ii) follows because $x_{i_{l}} y_{i_{k}}$ is an edge in $K_{n, n} \subset G$ for all $l=1, \cdots, n$.

There are also at least two nice characterizations of Cohen-Macaulay bipartite graphs.

Theorem 2.5 ([5, Theorem 3.4]). Let $G$ be a bipartite graph without isolated vertices. Then $G$ is Cohen-Macaulay if and only if there is a pure ordering $V_{1}=$ $\left\{x_{1}, \cdots, x_{n}\right\}$ and $V_{2}=\left\{y_{1}, \cdots, y_{n}\right\}$ of vertices of $G$ such that $x_{i} y_{j}$ being in $G$ implies $i \leq j$.

The ordering in Theorem 2.5 is called a Macaulay order of vertices of $G$.

Proposition 2.6 ([1, Proposition 4.8]). Let $G$ be a bipartite graph. Then $G$ is Cohen-Macaulay if and only if $G$ has a cross-free pure order.

Remark 2.7. Both characterizations of Cohen-Macaulay bipartite graphs are purely combinatorial (Theorem 2.3 together with Theorem 2.5 or Proposition 2.6). These characterizations may be taken as combinatorial definitions of Cohen-Macaulayness for bipartite graphs. Therefore, for bipartite graphs, the $\mathrm{CM}_{t}$ property can also be stated in combinatorial terms.

Recall that a complex is Buchsbaum if and only if it is pure and the link of each vertex is Cohen-Macaulay 9. Thus, a graph is Buchsbaum if and only if $G$ is unmixed and for every vertex $v \in G, G \backslash N[v]$ is Cohen-Macaulay. For bipartite graphs there is a sharper result. Complete bipartite graphs are well known to be Buchsbaum (e.g., see [12, Proposition 2.3]). But indeed, the converse is also true.

Theorem 2.8 (see [1, Theorem 4.10] or [3, Theorem1.3]). Let $G$ be a bipartite graph. Then $G$ is Buchsbaum if and only if $G$ is a complete bipartite graph $K_{n, n}$ for some $n \geq 2$, or $G$ is Cohen-Macaulay. 


\section{Joins of $\mathrm{CM}_{t}$ COMPlexes And DisJoint unions of $\mathrm{CM}_{t}$ GRAPHS}

It is known that the join of two complexes is Cohen-Macaulay if and only if they are both Cohen-Macaulay (see [8] and 2]). In fact, if the join of two complexes is Buchsbaum, it should indeed be Cohen-Macaulay [7, Theorem 2.6]. If $\Delta$ is a $\mathrm{CM}_{r}$ complex of dimension $d-1$ and $\Delta^{\prime}$ is a $\mathrm{CM}_{r^{\prime}}$ complex of dimension $d^{\prime}-1$, then their join $\Delta * \Delta^{\prime}$ is a $\mathrm{CM}_{t}$ complex where $t=\max \left\{d+r^{\prime}, d^{\prime}+r\right\}$ [4, Proposition 2.10]. However, if one of the complexes is Cohen-Macaulay, this result could be strengthened. Below we combine this with relevant known results.

Theorem 3.1. Let $\Delta$ and $\Delta^{\prime}$ be two complexes of dimensions $d-1$ and $d^{\prime}-1$, respectively. Then

(i) The join complex $\Delta * \Delta^{\prime}$ is Cohen-Macaulay if and only if both $\Delta$ and $\Delta^{\prime}$ are so.

(ii) If $\Delta$ is Cohen-Macaulay and $\Delta^{\prime}$ is $C M_{r^{\prime}}$ for some $r^{\prime} \geq 1$, then $\Delta * \Delta^{\prime}$ is $C M_{d+r^{\prime}}$ (independent of $d^{\prime}$ ). This is sharp, i.e., if $\Delta^{\prime}$ is not $C M_{r^{\prime}-1}$, then $\Delta * \Delta^{\prime}$ is not $C M_{d+r^{\prime}-1}$. In particular, a cone on $\Delta^{\prime}$ is $C M_{r^{\prime}+1}$.

(iii) If $\Delta$ is $C M_{r}$ and $\Delta^{\prime}$ is $C M_{r^{\prime}}$ for some $r, r^{\prime} \geq 1$, then $\Delta * \Delta^{\prime}$ is $C M_{t}$ where $t=\max \left\{d+r^{\prime}, d^{\prime}+r\right\}$. Conversely, if $\Delta * \Delta^{\prime}$ is $C M_{t}$, then $\Delta$ is $C M_{t-d^{\prime}}$ and $\Delta^{\prime}$ is $C M_{t-d}$.

Proof. The statement in (i) is proved by Sava [8] and Fröberg [2]. The assertion (iii) is proved in [4, Theorem 2.10]. We prove (ii) using induction on $d+r^{\prime} \geq 2$. Let $d+r^{\prime}=2$, i.e., $d=1$ and $r^{\prime}=1$. Then $\Delta=\{v\}$ is a singleton. Thus $\operatorname{link}_{\Delta * \Delta^{\prime}}(v)=\Delta^{\prime}$, which is $\mathrm{CM}_{1}$. For $v \in \Delta^{\prime}, \operatorname{link}_{\Delta * \Delta^{\prime}}(v)=\Delta * \operatorname{link}_{\Delta^{\prime}}(v)$, which is Cohen-Macaulay by (i). Thus by Lemma 2.1, $\Delta * \Delta^{\prime}$ is $\mathrm{CM}_{2}$. Now let $d+r^{\prime} \geq 2$. Let $v \in \Delta$. Then, $\operatorname{link}_{\Delta * \Delta^{\prime}}(v)=\operatorname{link}_{\Delta}(v) * \Delta^{\prime}$. But $\operatorname{link}_{\Delta}(v)$ is Cohen-Macaulay of dimension less than $d-1$, and $\Delta^{\prime}$ is $\mathrm{CM}_{r^{\prime}}$. Thus by induction hypothesis, $\operatorname{link}_{\Delta * \Delta^{\prime}}(v)$ is $\mathrm{CM}_{d-1+r^{\prime}}$. If $v \in \Delta^{\prime}$, then $\operatorname{link}_{\Delta * \Delta^{\prime}}(v)=\Delta^{\prime} * \operatorname{link}_{\Delta^{\prime}}(v)$. But $\operatorname{link}_{\Delta^{\prime}}(v)$ is $\mathrm{CM}_{r^{\prime}-1}$ and hence $\operatorname{link}_{\Delta * \Delta^{\prime}}(v)$ is again $\mathrm{CM}_{d+r^{\prime}-1}$. Therefore, $\Delta * \Delta^{\prime}$ is $\mathrm{CM}_{d+r^{\prime}}$. To prove that this result is sharp, proceed by induction on $d \geq 1$. Indeed, in this case, for any $v \in \Delta$, $\operatorname{link}_{\Delta}(v)$ has dimension less than $d-1$ and hence by induction hypothesis, $\operatorname{link}_{\Delta * \Delta^{\prime}}(v)=\operatorname{link}_{\Delta}(v) * \Delta$ is not $\mathrm{CM}_{d+r^{\prime}-2}$. Therefore, $\Delta * \Delta^{\prime}$ is not $\mathrm{CM}_{d+r^{\prime}-1}$

Let $G \sqcup G^{\prime}$ denote the disjoint union of graphs $G$ and $G^{\prime}$. By the fact that $\operatorname{Ind}\left(G \sqcup G^{\prime}\right)=\operatorname{Ind}(G) * \operatorname{Ind}\left(G^{\prime}\right)$, the counterpart of Theorem 3.1 for graphs will be the following.

Theorem 3.2. Let $G$ and $G^{\prime}$ be two graphs on disjoint sets of vertices and of dimensions $d-1$ and $d^{\prime}-1$, respectively. Then

(i) The graph $G \sqcup G^{\prime}$ is Cohen-Macaulay if and only if both $G$ and $G^{\prime}$ are so.

(ii) If $G$ is Cohen-Macaulay and $G^{\prime}$ is $C M_{r^{\prime}}$ for some $r^{\prime} \geq 1$, then $G \sqcup G^{\prime}$ is $C M_{d+r^{\prime}}$. If $G^{\prime}$ is not $C M_{r^{\prime}-1}$, then $G \sqcup G^{\prime}$ is not $C M_{d+r^{\prime}-1}$.

(iii) If $G$ is $C M_{r}$ and $G^{\prime}$ is $C M_{r^{\prime}}$ for some $r, r^{\prime} \geq 1$, then $G \sqcup G^{\prime}$ is $C M_{t}$ where $t=\max \left\{d+r^{\prime}, d^{\prime}+r\right\}$. Conversely, if $G \sqcup G^{\prime}$ is $C M_{t}$, then $G$ is $C M_{t-d^{\prime}}$ and $G^{\prime}$ is $C M_{t-d}$.

\section{Two Characterizations of Bipartite $\mathrm{CM}_{t}$ Graphs}

We now restrict to the case of bipartite graphs. Since Cohen-Macaulay bipartite graphs are characterized by Herzog and Hibi [5. Theorem 3.4], and also in 
a different version by Cook and Nagel [1, Proposition 4.8], we consider the nonCohen-Macaulay case.

Theorem 4.1. Let $G$ be an unmixed bipartite graph of dimensions $d-1$. Let $K_{n, n}$, with $n \geq 2$, be a maximal complete bipartite subgraph of $G$ of minimum dimension. Then $G$ is $C M_{d-n+1}$ but it is not $C M_{d-n}$.

Proof. We prove both assertions by induction on $d \geq 2$. If $d=2$, then $G=K_{2,2}$ which is $\mathrm{CM}_{1}$ but it is not Cohen-Macaulay. Assume that $d>2$. We show that for every $v \in G, G \backslash N[v]$ is $\mathrm{CM}_{n-d}$ and for some $v \in G$ it is not $\mathrm{CM}_{n-d-1}$. Let $\left(\left\{x_{1}, \cdots, x_{d}\right\},\left\{y_{1}, \cdots, y_{d}\right\}\right)$ be a pure order of $G$. Let $x_{i}$ be a vertex of some maximal bipartite subgraph $K_{m, m}$ with $m \geq n$. Then $G \backslash N\left[x_{i}\right]$ is a disjoint union of $c \geq m-1$ isolated vertices and an unmixed bipartite graph $H$ of dimension $d-c-2$. The graph $H$ is unmixed because $\operatorname{Ind}\left(G \backslash N\left[x_{i}\right]\right)=\operatorname{link}_{x_{i}}(\operatorname{Ind}(G))$, and any link of a pure complex is pure. But $G \backslash N\left[x_{i}\right]=\left\{x_{i_{1}}, \cdots, x_{i_{c}}\right\} \sqcup H$ is unmixed if and only if $H$ is so. Observe that if $y_{j_{0}}$ is a vertex of a maximal bipartite subgraph of $G$ and $y_{j_{0}} \in N\left[x_{i}\right]$, then by Lemma 2.4 all $y_{j}$ vertices of this subgraph belong to $N\left[x_{i}\right]$. Thus if $H$ has no crosses, by Proposition 2.6 it is Cohen-Macaulay. Otherwise, the minimum dimension of maximal complete bipartite subgraphs of $H$ will not be less than the minimum dimension of such subgraphs in $G$. Hence by the induction hypothesis, $H$ is $\mathrm{CM}_{d-c-n}$ and by Theorem [3.2(ii), $G \backslash N\left[x_{i}\right]$ is $\mathrm{CM}_{n-d}$. If $x_{i}$ does not belong to any maximal bipartite subgraph of $G$ of positive dimension, then $G \backslash N\left[x_{i}\right]$ is a disjoint union of $c \geq 0$ isolated vertices and an unmixed bipartite graph $H$ of dimension $d-c-2$. Hence $H$ is $\mathrm{CM}_{d-c-n}$ and by Theorem $\left[3.2\left(\right.\right.$ ii),$G \backslash N\left[x_{i}\right]$ is $\mathrm{CM}_{d-n}$. A similar argument reveals that for any $y_{i} \in G$, the graph $G \backslash N\left[y_{i}\right]$ is $\mathrm{CM}_{d-n}$. Therefore, by Lemma 2.2 $G$ is $\mathrm{CM}_{d-n+1}$. We now proceed with the induction step to show that this result is sharp. Let $d>2$ and let $K_{n, n}, n \geq 2$, be a maximal bipartite subgraph of $G$ of minimum dimension. Take $x_{i} \in G \backslash K_{n, n}$. First assume that $x_{i}$ is not adjacent to any vertex in $K_{n, n}$ and consider $G \backslash N\left[x_{i}\right]$. Let $G \backslash N\left[x_{i}\right]$ be the disjoint union of $c \geq 0$ isolated vertices and an unmixed bipartite graph $H$ of dimension $d-c-2$. Then $H$ contains $K_{n, n}$ and hence by induction hypothesis, $H$ is sharp $\mathrm{CM}_{d-c-n}$ and $G \backslash N\left[x_{i}\right]$ is sharp $\mathrm{CM}_{d-n}$. Therefore, $G$ cannot be $\mathrm{CM}_{d-n}$. Now assume that $x_{i} y_{j} \in G$ for some $j$ with $y_{j} \in K_{n, n}$. Then by purity of the order, all $y_{k} \in K_{n, n}$ is adjacent to $x_{i}$. But then $y_{i}$ is not adjacent to any vertex of $K_{n, n}$, because otherwise, $K_{n, n}$ will not be maximal. In this case, consider $G \backslash N\left[y_{i}\right]$ and proceed in a way similar to the previous case.

As a second characterization of bipartite $\mathrm{CM}_{t}$ graphs, we show that any $\mathrm{CM}_{t}$ graph is obtained from a Cohen-Macaulay graph $H$ by replacing the perfect matching edges of $H$ by complete bipartite graphs. This statement will be more precise in the next theorem. But first we provide a definition and a lemma.

Definition 4.2. Let $H$ be an unmixed bipartite graph with pure order

$$
\left(\left\{x_{1}, \cdots, x_{r}\right\},\left\{y_{1}, \cdots, y_{r}\right\}\right) .
$$

For a fixed $i$, by replacing the edge $x_{i} y_{i} \in H$ with a complete bipartite graph

$$
K_{n_{i}, n_{i}}=\left\{x_{i 1}, \cdots, x_{i n_{i}}\right\} \times\left\{y_{i 1}, \cdots, y_{i n_{i}}\right\}
$$


we mean a bipartite graph $H^{\prime}$ with vertex set

$$
\begin{aligned}
\left(\left\{x_{1}, \cdots, x_{i-1}, x_{i 1}, \cdots, x_{i n_{i}}, x_{i+1}, \cdots, x_{r}\right\}\right. & \\
& \left.\left\{y_{1}, \cdots, y_{i-1}, y_{i 1}, \cdots, y_{i n_{i}}, y_{i+1}, \cdots, y_{r}\right\}\right),
\end{aligned}
$$

preserving all adjacencies, i.e.,

(i) $x_{s} y_{t} \in H^{\prime}$ for all $s, t \neq i$ if and only if $x_{t} y_{s} \in H$,

(ii) $x_{i_{k}} y_{j} \in H^{\prime}$ for all $k$ if and only if $x_{i} y_{j} \in H$,

(iii) $x_{j} y_{i_{k}} \in H^{\prime}$ for all $k$ if and only if $x_{j} y_{i} \in H$.

Lemma 4.3. Let $G$ be an unmixed bipartite graph with pure order on the vertex set $V(G)=V \cup W$ where $V=\left\{x_{1}, \cdots, x_{d}\right\}$ and $W=\left\{y_{1}, \cdots, y_{d}\right\}$. Let $n_{1}, \cdots, n_{d}$ be any positive integers. Let $G^{\prime}=G\left(n_{1}, \cdots, n_{d}\right)$ be the graph obtained by replacing each edge $x_{i} y_{i}$ with the complete bipartite graph $K_{n_{i}, n_{i}}=\left\{x_{i 1}, \cdots, x_{i n_{i}}\right\} \times$ $\left\{y_{i 1}, \cdots, y_{i n_{i}}\right\}$ for all $i=1, \ldots, d$. Then $G^{\prime}$ is also unmixed.

Proof. Observe that

$$
V\left(G^{\prime}\right)=\left(\left\{x_{11}, \cdots, x_{1 n_{1}}, \cdots, x_{d 1}, \cdots, x_{d n_{d}}\right\},\left\{y_{11}, \cdots, y_{1 n_{1}}, \cdots, y_{d 1}, \cdots, y_{d n_{d}}\right\}\right)
$$

is a pure order of $G^{\prime}$. In fact, for all $i, r, x_{i r} y_{i r} \in G^{\prime}$. Also if $x_{i r} y_{j s} \in G^{\prime}$ and $x_{j s} y_{k t} \in G^{\prime}$, then $x_{i} y_{j} \in G$ and $x_{j} y_{k} \in G$, and hence, $x_{i} y_{k} \in G$. Thus by the construction of $G^{\prime}, x_{i r} y_{k t} \in G^{\prime}$.

Theorem 4.4. Let $G$ be a Cohen-Macaulay bipartite graph with a Macaulay order on the vertex set $V(G)=V \cup W$ where $V=\left\{x_{1}, \cdots, x_{d}\right\}$ and $W=\left\{y_{1}, \cdots, y_{d}\right\}$. Let $n_{1}, \cdots, n_{d}$ be any positive integers with $n_{i} \geq 2$ for at least one value of $i$. Let $G^{\prime}=G\left(n_{1}, \cdots, n_{d}\right)$ be the graph obtained by replacing each edge $x_{i} y_{i}$ with the complete bipartite graph $K_{n_{i}, n_{i}}$ for all $i=1, \ldots, d$. Let $n_{i_{0}}=\min \left\{n_{i}>1: i=\right.$ $1, \cdots, d\}, n=\sum_{i=1}^{d} n_{i}$. Then $G^{\prime}$ is exclusively a $C M_{n-n_{i_{0}}+1}$ graph. Furthermore, any bipartite $C M_{t}$ graph is obtained by such a replacement of complete bipartite graphs in a unique bipartite Cohen-Macaulay graph.

Proof. The first claim follows by Lemma 4.3 and Theorem 4.1. We settle the second claim. Let $G$ be a bipartite $\mathrm{CM}_{t}$ graph with a pure order of vertices. Let $K_{n_{1}, n_{1}}, \cdots, K_{n_{d}, n_{d}}$ be the maximal bipartite subgraphs of $G$, where $n_{i} \geq 1$ for all $i$. Observe that, by maximality, these complete subgraphs of $G$ are disjoint. Choose one edge $x_{i 1} y_{i 1}$ from each subgraph $K_{n_{i}, n_{i}}$ for all $i=1, \cdots, d$. Let $H$ be the induced subgraph of $G$ on the vertex set $\left(\left\{x_{11}, \cdots, x_{d 1}\right\},\left\{y_{11}, \cdots, y_{d 1}\right\}\right)$. By Lemma 2.4. $H$ is independent of the choice of particular edge $x_{i 1} y_{i 1}$ from $K_{n_{i}, n_{i}}$ and hence $H$ is unique. Since the ordering of vertices of $G$ is a pure order, its restriction to $H$ is also pure. Thus, $H$ is an unmixed bipartite graph. But by maximality of the complete bipartite subgraphs $K_{n_{i}, n_{i}}$, and the construction of $H$, it is cross-free. Therefore, by Proposition 2.6. $H$ is Cohen-Macaulay. Now replace any edge $x_{i 1} y_{i 1}$ in $H$ with $K_{n_{i}, n_{i}}$ for all $i=1, \cdots, d$, preserving all other adjacencies. Let $H^{\prime}$ be the resulting graph. Then by the construction, $G=H^{\prime}$, as required.

Remark 4.5. Let $H$ be a bipartite Cohen-Macaulay graph and let $G=H^{\prime}$ be a bipartite $\mathrm{CM}_{t}$ graph obtained from $H$ by the replacing process described above. Assume that $G$ is is not $\mathrm{CM}_{t-1}$ and $t \geq 2$. Using the results of this section, the following observations are immediate. 


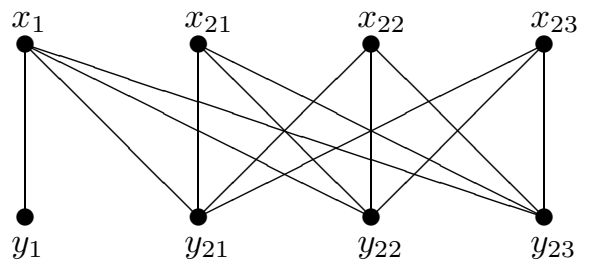

Figure 1

First of all, $1 \leq \operatorname{dim} H \leq t-1$. Because if $\operatorname{dim} H \geq t$ and we replace just one edge with $K_{n, n}$ where $n \geq 2$, then $G$ is strictly $\mathrm{CM}_{r}$ with $r \geq t+1$. On the other hand, if $\operatorname{dim} H=0$, then $G$ is $\mathrm{CM}_{1}$.

If $\operatorname{dim} H=t-1$, then only one edge can be replaced with $K_{n, n}$ where $n \geq 2$. Because replacing at least two edges with $K_{n, n}$ 's, $n \geq 2, G$ will be strictly $\mathrm{CM}_{r}$ where $r \geq t+1$.

If $\operatorname{dim} H=t-1$, for replacing just one edge with $K_{n, n}, n$ can be arbitrary and hence $G$ will be of dimension $n+t-2$.

If $\operatorname{dim} H \leq t-2$, the number of replacements should be at least 2. Again because if we replace one edge with $K_{n, n}, n \geq 2$, then $G$ would be $\mathrm{CM}_{r}$ for $r \leq t-1$.

When $\operatorname{dim} H \leq t-2$, the maximum number of replacements of edges with $K_{n, n}$, $n \geq 2$, is at most $t-\operatorname{dim} H$ which may occur replacing $K_{2,2}$ 's.

For $\operatorname{dim} H \leq t-2$, the maximum size of $K_{n, n}$ to be used for replacements is also $n=t-\operatorname{dim} H$ which may occur when we have two replacements.

Using these remarks we may easily distinguish all bipartite $\mathrm{CM}_{t}$ graphs for $t=2,3,4$.

Example 4.6. Bipartite $\mathrm{CM}_{2}$ graphs which are not Buchsbaum. Using the notation of Remark 4.5 we have $\operatorname{dim} H=1$. There are just two nonisomorphic bipartite Cohen-Macaulay graphs of dimension one. By the replacing process, they produce two types of bipartite $\mathrm{CM}_{2}$ graphs which are not Buchsbaum. They are of arbitrary dimensions. More precisely, one such graph is the disjoint union of an edge $x_{1} y_{1}$ with $K_{n_{2}, n_{2}}=\left\{x_{21}, \cdots, x_{2 n_{2}}\right\} \times\left\{y_{21}, \cdots, y_{2 n_{2}}\right\}, n_{2} \geq 2$, and the other one consists of the first graph together with the edges $x_{1} y_{2 i}$ for all $i=1, \cdots, n_{2}$. The second graph with $n_{2}=3$ could be depicted in Figure 1 .

Example 4.7. Bipartite $\mathrm{CM}_{3}$ graphs which are not $\mathrm{CM}_{2}$. For these graphs $\operatorname{dim} H=1,2$.

If $\operatorname{dim} H=1$, by Example 4.6. there are just two bipartite $\mathrm{CM}_{3}$ graphs obtained by replacing two edges of a perfect matching by $K_{2,2}$ 's. In this case, $\operatorname{dim} G=3$ (see Figure 2 and Figure 3).

If $\operatorname{dim} H=2$, then there are 4 non-isomorphic bipartite Cohen-Macaulay graphs of dimension 2. By replacing one perfect matching edge with $K_{n, n}$ of arbitrary size in each Cohen-Macaulay graph, they produce 7 types of bipartite $\mathrm{CM}_{3}$ graphs which are not $\mathrm{CM}_{2}$. Note that depending on the choice of the edge to be replaced in each case, we may get nonisomorphic bipartite graphs. In this case $\operatorname{dim} G=n+1$.

Example 4.8. Bipartite $\mathrm{CM}_{4}$ graphs which are not $\mathrm{CM}_{3}$. For these graphs $\operatorname{dim} H=1,2,3$. 

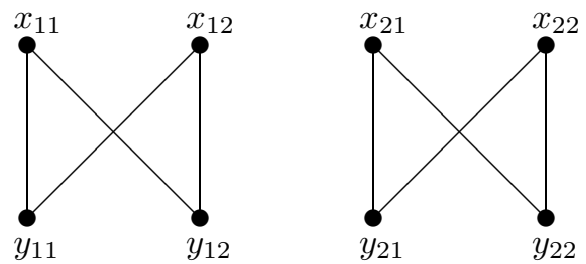

FiguRE 2

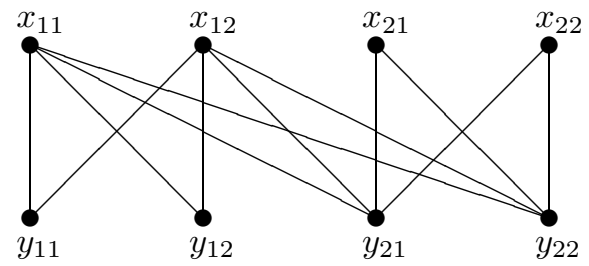

FIGURE 3

If $\operatorname{dim} H=1$, there are two bipartite $\mathrm{CM}_{4}$ graphs obtained by replacing two edges of a perfect matching by $K_{3,3}$ 's. And, similarly, there are two others obtained by replacing one edge with $K_{2,2}$ and another edge with $K_{3,3}$. In both cases, $\operatorname{dim} G=5$.

If $\operatorname{dim} H=2$, then while there are 4 nonisomorphic bipartite Cohen-Macaulay graphs of dimension 2, by replacing two perfect matching edges with $K_{2,2}$ 's in each Cohen-Macaulay graph, they produce 7 bipartite $\mathrm{CM}_{4}$ graphs which are not $\mathrm{CM}_{3}$. They all have dimension 4 .

If $\operatorname{dim} H=3$, then there are 10 nonisomorphic bipartite Cohen-Macaulay graphs of dimension 3. Replacing one perfect matching edge with $K_{n, n}, n \geq 2$, in each Cohen-Macaulay graph, they produce 25 bipartite $\mathrm{CM}_{4}$ graphs which are not $\mathrm{CM}_{3}$. They all have dimension $n+2$. Out of all 36 bipartite $\mathrm{CM}_{4}$ graphs, 21 graphs are connected.

\section{ACKNOWLEDGMENTS}

The authors would like to thank J. Herzog and U. Nagel for some fruitful discussions on the subject of this paper. This work has been supported by the Center for International Studies \& Collaboration (CISSC) and the French Embassy in Tehran in the framework of the Gundishapur project 27462PL on the Homological and Combinatorial Aspects of Commutative Algebra and Algebraic Geometry. The research of the second author was supported in part by a grant from IPM (No. 92130422). The third author has been partially supported by research grant no. 4/1/6103011 of the University of Tehran.

\section{REFERENCES}

[1] David Cook II and Uwe Nagel, Cohen-Macaulay graphs and face vectors of flag complexes, SIAM J. Discrete Math. 26 (2012), no. 1, 89-101, DOI 10.1137/100818170. MR2902635

[2] Ralf Fröberg, A note on the Stanley-Reisner ring of a join and of a suspension, Manuscripta Math. 60 (1988), no. 1, 89-91, DOI 10.1007/BF01168149. MR.920761 (89b:52013)

[3] Hassan Haghighi, Siamak Yassemi, and Rahim Zaare-Nahandi, Bipartite $S_{2}$ graphs are Cohen-Macaulay, Bull. Math. Soc. Sci. Math. Roumanie (N.S.) 53(101) (2010), no. 2, 125132. MR2667973 (2011g:13044) 
[4] Hassan Haghighi, Siamak Yassemi, and Rahim Zaare-Nahandi, A generalization of $k$-CohenMacaulay simplicial complexes, Ark. Mat. 50 (2012), no. 2, 279-290, DOI 10.1007/s11512010-0136-y. MR2961323

[5] Jürgen Herzog and Takayuki Hibi, Distributive lattices, bipartite graphs and Alexander duality, J. Algebraic Combin. 22 (2005), no. 3, 289-302, DOI 10.1007/s10801-005-4528-1. MR2181367 (2006h:06004)

[6] Ezra Miller, Isabella Novik, and Ed Swartz, Face rings of simplicial complexes with singularities, Math. Ann. 351 (2011), no. 4, 857-875, DOI 10.1007/s00208-010-0620-5. MR2854116 (2012j:13040)

[7] Hossein Sabzrou, Massoud Tousi, and Siamak Yassemi, Simplicial join via tensor product, Manuscripta Math. 126 (2008), no. 2, 255-272, DOI 10.1007/s00229-008-0175-x. MR2403189 (2009c:13021)

[8] Corina Sava, On the Stanley-Reisner ring of a join, An. Ştiinţ. Univ. Al. I. Cuza Iaşi Secţ. I a Mat. 31 (1985), no. 2, 145-148. MR858053 (87k:13039)

[9] Peter Schenzel, On the number of faces of simplicial complexes and the purity of Frobenius, Math. Z. 178 (1981), no. 1, 125-142, DOI 10.1007/BF01218376. MR627099 (82k:13022)

[10] Richard P. Stanley, Combinatorics and commutative algebra, 2nd ed., Progress in Mathematics, vol. 41, Birkhäuser Boston Inc., Boston, MA, 1996. MR1453579 (98h:05001)

[11] Rafael H. Villarreal, Unmixed bipartite graphs (English, with English and Spanish summaries), Rev. Colombiana Mat. 41 (2007), no. 2, 393-395. MR2585907 (2011c:05283)

[12] Rahim Zaare-Nahandi and Rashid Zaare-Nahandi, The minimal free resolution of a class of square-free monomial ideals, J. Pure Appl. Algebra 189 (2004), no. 1-3, 263-278, DOI 10.1016/j.jpaa.2003.10.026. MR2038574 (2005e:13021)

Department of Mathematics, K. N. Toosi University of Technology, Tehran, Iran

E-mail address: haghighi@kntu.ac.ir

School of Mathematics, Statistics \& Computer Science, University of Tehran, Tehran Iran - And - School of Mathematics, Institute for Research in Fundamental SCIEnCES (IPM), P.O. Box 19395-5746, Tehran, Iran

E-mail address: yassemi@ipm.ir

E-mail address: yassemi@ut.ac.ir

School of Mathematics, Statistics \& Computer Science, University of Tehran, TEHran IRAN

E-mail address: rahimzn@ut.ac.ir 